\title{
La leishmaniose en Grèce.
}

\author{
Résultats d'une enquête entomologique \\ effectuée en juin 1977.
}

\author{
par N. LEgER, A. SARATSIOTIS, B. PESSON et P. LEgER \\ Laboratoire de Parasitologie de l'U.E.R. de Pharmacie de Reims, F 51096 Reims \\ et Laboratoire de Parasitologie de l'Institut \\ de Microbiologie vétérinaire d'Athènes, Grèce.
}

\section{Résumé.}

En relation avec la recrudescence actuelle de la leishmaniose en Grèce, les auteurs ont entrepris une enquête entomologique dans le but de circonscrire les foyers de transmission. Un premier piégeage effectué en juin 1977 en Grèce continentale a permis de récolter 6633 phlébotomes, avec $21 \%$ de $P$. major et $20 \%$ de $P$. perfiliewi. Les captures sont analysées par espèces avec commentaires épidémiologiques et systématiques.

\section{Summary.}

Leishmaniasis in Greece. Results of an entomological survey during June 1977.

In relation with the recent outbreak of leishmaniasis in Greece, the authors undertook entomological investigations for delimiting the foci of transmission. A first survey, during june 1977, in continental Greece, gave 6633 sandflies with $21 \%$ of $P$. major and $20 \%$ of $P$. perfiliewi. Captures are analysed for each species with systematic and epidemiological comments.

La Grèce est un foyer classique de leishmaniose, aussi bien cutanée que viscérale et canine.

A partir de 1935 la campagne antirabique, puis les événements de 1940-1945 entraînent une forte diminution de l'effectif canin.

Accepté le 7 décembre 1978. 
D'autre part à la suite des pulvérisations de D.D.T. effectuées à partir de 1946 dans le cadre de la lutte antipalustre, on observe une diminution spectaculaire des populations de phlébotomes dans toutes les zones traitées (Hertig, 1949).

C'est à l'action combinée de ces deux facteurs qu'il convient de rapporter la diminution concomitante de l'endémie leishmanienne, particulièrement nette pour le kala-azar.

Mais du fait de l'arrêt des pulvérisations consécutif à l'éradication du paludisme et de l'augmentation constante de la densité canine, les effectifs de phlébotomes semblent s'être actuellement reconstitués et la prévalence de la leishmaniose canine atteint dans certaines régions (Attique notamment) un niveau alarmant.

Parallèlement on voit augmenter le nombre des cas de leishmaniose humaine, viscérale surtout, relevés chaque année (fig. 1 et tableau 1).

Tableau I. - Cas humains recensés en Grèce de 1951 à 1975. (entre parenthèses les cas observés à Athènes).

\begin{tabular}{|c|c|c|}
\hline Années & Leishmaniose cutanée & Leishmaniose viscérale \\
\hline 1951-1955 ..................... & $17(0)$ & $151 \quad(0)$ \\
\hline $1956-1960 \ldots \ldots \ldots \ldots \ldots \ldots \ldots \ldots$ & $12(0)$ & $168 \quad(0)$ \\
\hline 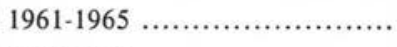 & $28(0)$ & $310 \quad(0)$ \\
\hline 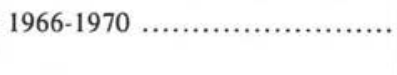 & $\begin{array}{c}167(0) \\
(159 \text { cas à Zanthe) }\end{array}$ & $307(125)$ \\
\hline 1970-1975 ..................... & $47(7)$ & $228(112)$ \\
\hline
\end{tabular}

Dans le cadre d'un bilan de la situation en Grèce continentale nous avons entrepris en juin 1977 une enquête entomologique dans le but de circonscrire les foyers à haut risque.

\section{I. - Résumé des travaux antérieurs}

Mis à part quelques mentions isolées d'espèces (Adler et Théodor, 1931-1935), c'est à Caminopetros $(1934,1935)$ et à Parrot $(1935,1936)$ que nous devons les premiers inventaires des Phlébotomes de Grèce, limités, outre Athènes et l'Attique, à quelques localités du Péloponèse et à la Crète (fig. 2).

En 1938, dans un travail qui reste encore en bien des points exemplaire, Adler, Théodor et Witenberg étudient le foyer leishmanien de Crète. Ils signalent des espèces de Phlébotomes nouvelles pour la Grèce et individualisent à La Canée, deux complexes épidémiologiques : «Leishmania infantum - Phlebotomus major 》 et « Leishmania tropica - Phlebotomus sergenti ».

En 1939 une enquête effectuće en Macédoine (Hadjinicolaou, 1958) vient compléter les données chorologiques précédentes. 


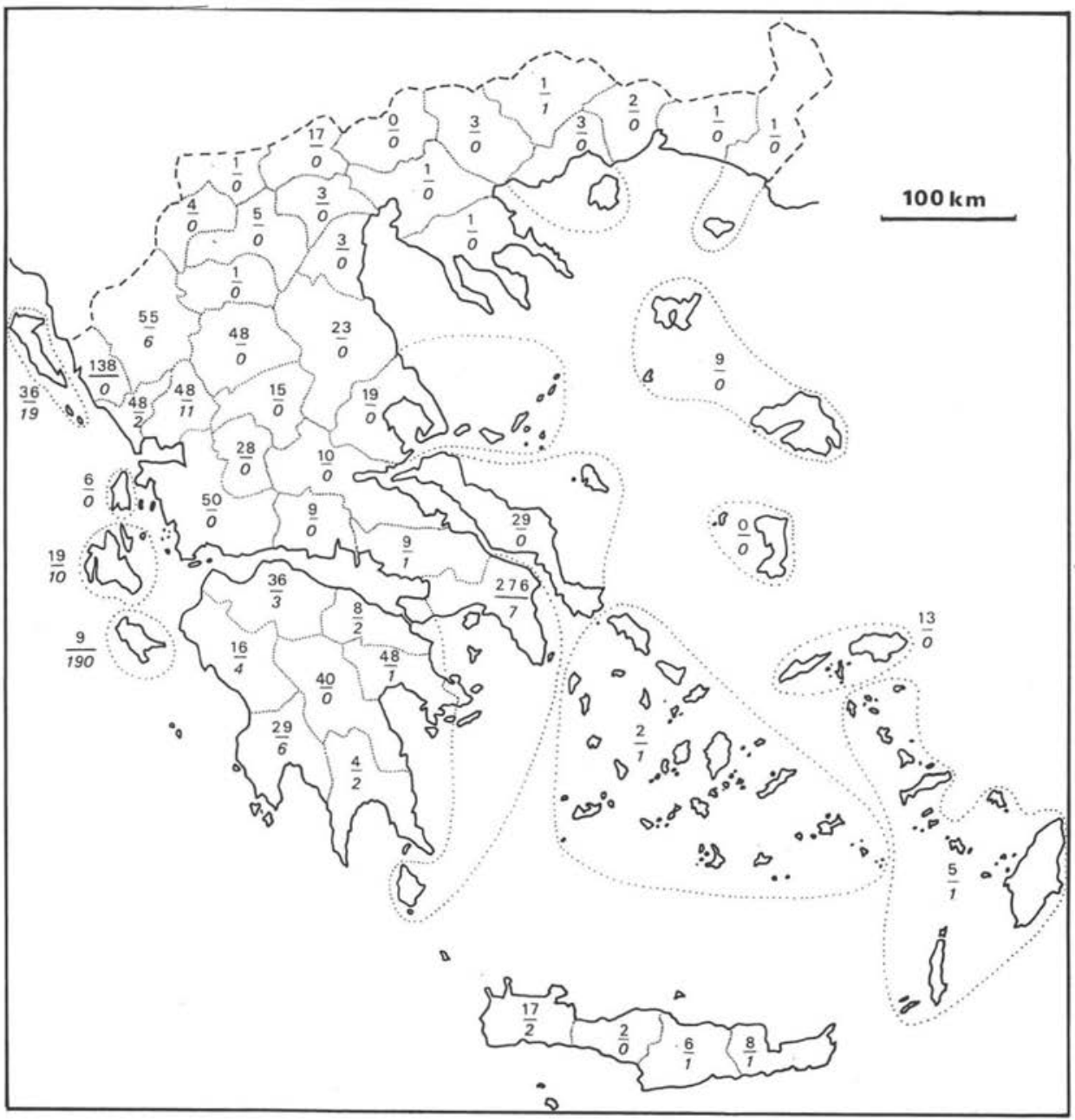

Fig. 1. - Répartition des cas humains de leishmaniose recensés en Grèce de 1951 à 1975. Caractères gras: 1. viscérale; caractères italiques: 1. cutanée (d'après A. Saratsiotis: non publié).

A partir de 1948 des chercheurs s'intéressent de nouveau aux Phlébotomes de Grèce dans le but surtout d'étudier les effets produits par le D.D.T. : enquêtes dans l'Attique et la Crète (Hertig, 1949) et dans diverses îles (Eubée, îles du golfe de Saronikos) ainsi que dans le district de Lakonia (Péloponèse) (Hadjinicolaou, 1958).

A noter qu'il s'agit toujours de captures ponctuelles, provenant le plus souvent de gîtes domestiques. 


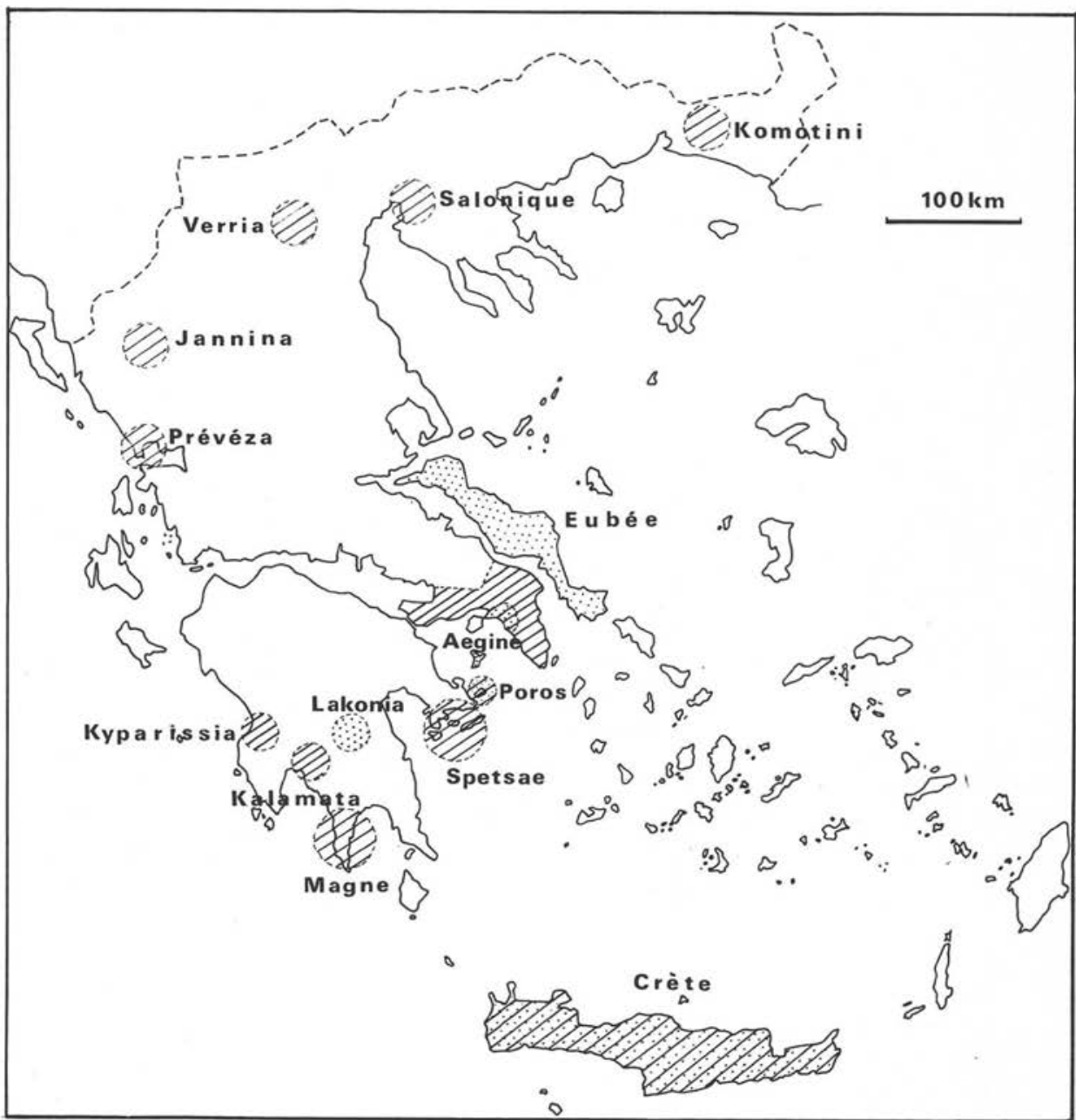

Fig. 2. - Précédentes enquêtes sur les Phlébotomes de Grèce. En hachuré : 1934-1939, avant le DDT (Parrot, Caminopetros, Adler et coll., Hadjinicolaou). En pointillé : 1949-1958, après le DDT (Hertig, Hadjinicolaou).

\section{II. - Choix et description de la zone étudiée}

Nos piégeages ont été effectués dans une région inexplorée en ce qui concerne les Phlébotomes (fig. 3).

Il s'agit d'une zone pratiquement indemne de leishmaniose cutanée mais où l'incidence du kala-azar est assez élevée (fig. 1). 


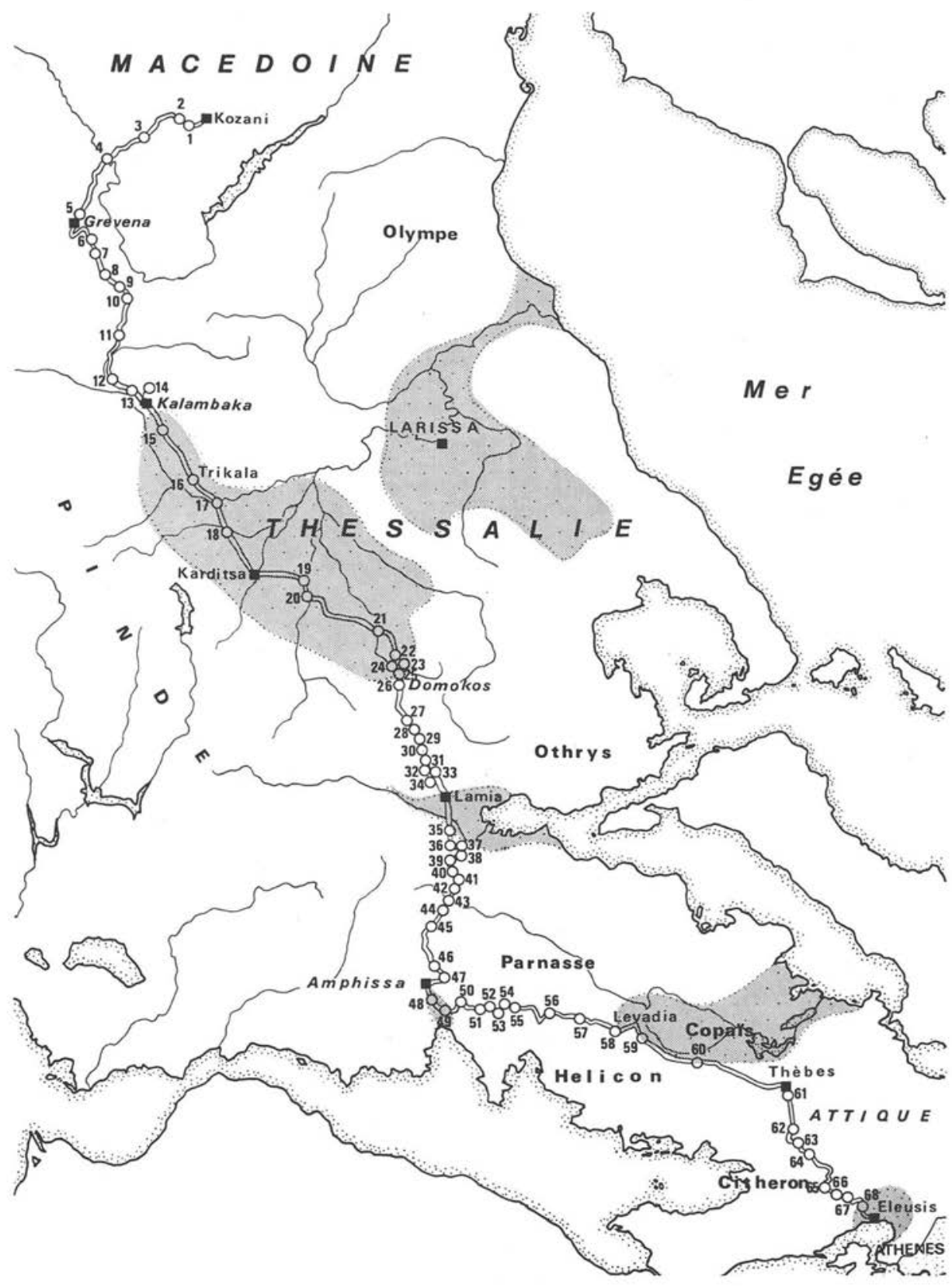

Fig. 3. - Région prospectée et itinéraire. 
L'échantillonnage des Phlébotomes a été réalisé à l'aide de piégeages stationnels le long d'un itinéraire (choisi en fonction des données recueillies sur l'origine des cas récents de leishmaniose canine) qui va de Kosani à Eleusis en passant par: Grevena Trikala - Karditsa - Lamia - Amphissa - Delphes - Thèbes.

Cet itinéraire-transect traverse successivement :

1) Les derniers contreforts sud du massif cristallin macédonien, bordés à l'ouest par les montagnes calcaires du Pinde auxquelles se rattachent les Météores au relief karstique caractéristique.

Cette zone présente un peuplement de Chênes verts et, aux altitudes plus élevées, de Chênes caducifoliés.

2) La plaine de Thessalie couverte de céréales et d'oliviers.

3) Une série de petits massifs de nature souvent karstique (Othrys, Parnasse, Hélicon, Cithéron) entrecoupés de vallées et de dépressions qui s'ouvrent soit sur le golfe de Lamia (vallée du Spercheios, Copaïs), soit sur le golfe de Corinthe (golfe de Krissa, baie d'Eleusis). Sur ces terrains calcaires on trouve un maquis de Chêne kermès (Quercus coccifera) associé au Cade (Juniperus oxycedrus) dans l'Othrys et au Cade, au Genévrier de Phénicie (Juniperus phoenicea) et au Pin d'Alep (Pinus halapensis) au sud de Lamia. Au-dessus de 700 m c'est la forêt d'Abies cephalonica.

\section{III. - Méthode d'échantillonnage.}

Nous avons utilisé la capture aux pièges adhésifs (Croset et coll., 1977) qui se prête bien aux études chorologiques quantitatives.

Sur notre itinéraire de $554 \mathrm{~km}$ nous avons réalisé 68 piégeages stationnels, soit en moyenne une station tous les $8 \mathrm{~km}$, en essayant d'explorer les divers types de biotopes sauvages, à l'exclusion presque complète des gîtes domestiques.

Le nombre de papiers mis en place dans chaque station est très variable, de 3 à 72 , et dépend du nombre des anfractuosités favorables à la pose des pièges.

Au total 2000 papiers ont été posés et 1567 ont pu être récupérés.

La mise en place des pièges a été effectuée du 6 au 9 juin et le relevé du 11 au 12 .

6633 phlébotomes ont été récoltés de cette façon.

\section{IV. - Analyse des résultats et commentaires sur les espèces}

D'après Hertig (1949) et Théodor (1958) 12 espèces de Phlébotomes sont connues de Grèce.

Nous avons retrouvé toutes ces espèces à l'exception de Phlebotomus mascitti Grassi 1908 (signalé seulement en Crète) et Sergentomyia theodori (Parrot 1942).

Nos captures sont données en détail dans le tableau II et en résumé dans le tableau III (S. bruchoni, signalé par Hertig $(1948)=S$. dentata dans notre tableau, cf. infra). 


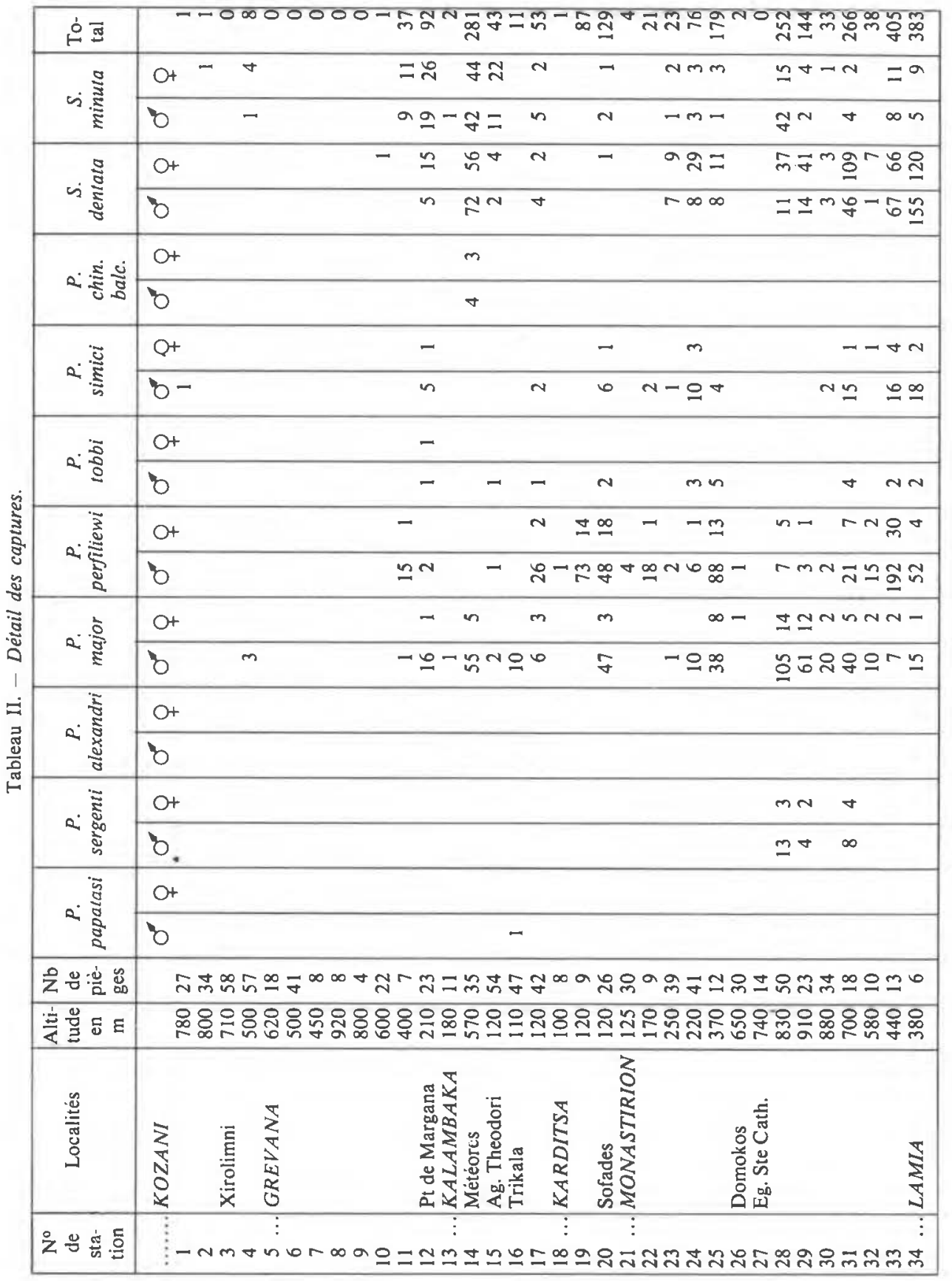

Annales de Parasitologie humaine et comparée (Paris), t. 54, $\mathrm{n}^{\circ} 1$. 


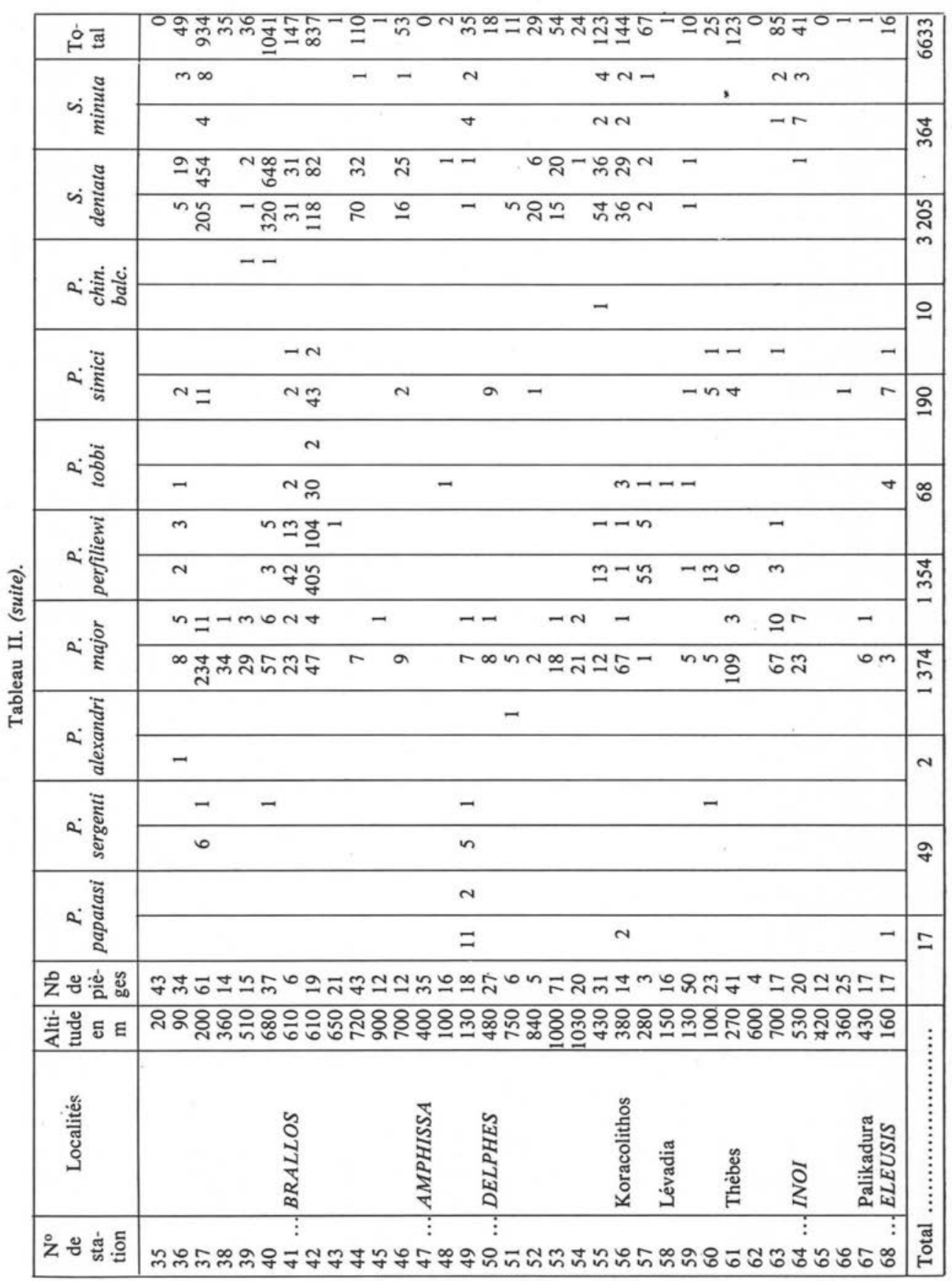


Tableau III. - Abondance relative des différentes espèces dans nos captures.

\begin{tabular}{|c|c|c|c|c|c|}
\hline Genres & Sous-genres & Espèces & \multicolumn{3}{|c|}{ Pourcentages } \\
\hline \multirow[t]{4}{*}{ Phlebotomus } & Phlebotomus & papatasi & 0,3 & 0,3 & \multirow{4}{*}{46} \\
\hline & Paraphlebotomus & $\begin{array}{l}\text { sergenti } \\
\text { alexandri }\end{array}$ & $\begin{array}{l}0,7 \\
0,03\end{array}$ & 0,73 & \\
\hline & Larroussius & $\begin{array}{l}\text { major syriacus } \\
\text { perfiliewi } \\
\text { tobbi }\end{array}$ & $\begin{array}{r}21 \\
20 \\
1\end{array}$ & 42 & \\
\hline & Adlerius & $\begin{array}{l}\text { simici } \\
\text { chin. balcanicus }\end{array}$ & $\begin{array}{l}3 \\
0,2\end{array}$ & 3,2 & \\
\hline Sergentomyia & & $\begin{array}{l}\text { dentata } \\
\text { minuta }\end{array}$ & $\begin{array}{r}48 \\
5\end{array}$ & \multicolumn{2}{|c|}{53} \\
\hline
\end{tabular}

\section{A. - Genre Phlebotomus :}

\section{$1^{\circ}$ Sous-genre Phlebotomus :}

- Phlebotomus papatasi Scopoli 1789 :

En désaccord avec les chiffres donnés par les autres auteurs, nous n'avons capturé que peu de $P$. papatasi. Ce résultat est sans doute dû aux faits que nous avons surtout piégé des biotopes sauvages (or on connaît l'anthropophilie accusée de cette espèce, du moins dans la région considérée) et que nos captures ont été effectuées tôt dans la saison, sans doute bien avant le maximum des sorties.

\section{$2^{\circ}$ Sous-genre Paraphlebotomus :}

- Phlebotomus sergenti Parrot 1917,

- et P. alexandri Sinton 1928 :

Vecteurs classiques de Leishmania tropica, ces deux espèces n'ont été capturées qu'à un petit nombre d'exemplaires, ce qui est en accord avec l'absence de leishmaniose cutanée dans la région.

\section{$3^{\circ}$ Sous-genre Larroussius :}

C'est à ce groupe, très souvent mis en cause dans la transmission de Leishmania infantum, qu'appartient la grande majorité des Phlebotomus capturés.

Dans la région prospectée, deux espèces : $P$. major et $P$. perfiliewi apparaissent comme hautement suspectes du fait de leur abondance.

- Phlebotomus major Annandale 1910:

Ce binôme recouvre un complexe d'espèces ou de sous-espèces dont la diagnose est souvent difficile en l'absence d'exemplaires de référence accessibles. Nous appuyant sur l'origine géographique et les critères de différenciation donnés par Perfiliew (1966) et Théodor (1958), nous avons identifié nos captures (fig. 4) comme $P$. major syriacus 


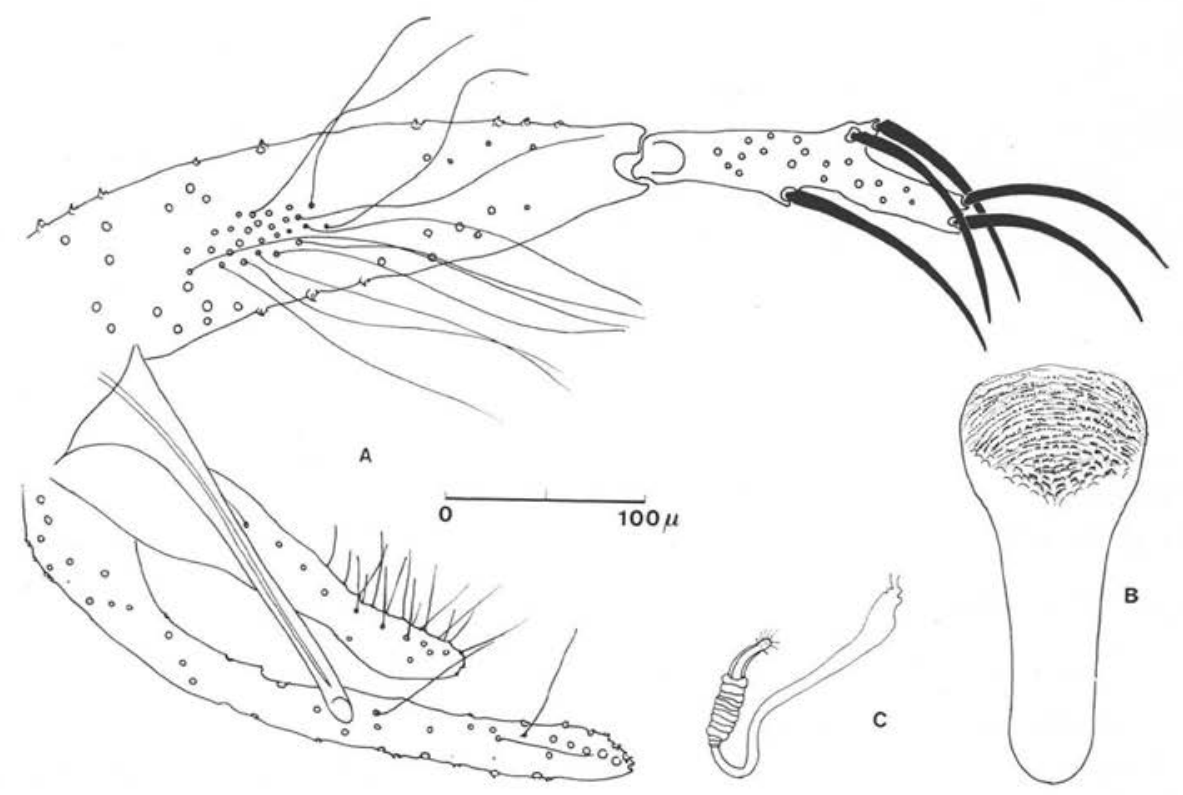

Fig. 4. - Phlebotomus major Syriacus $\delta^{\star}$ et $q$. A, genitalia; B, pharynx $q$; C, spermathèque.

Alder et Théodor 1931. Les mêmes auteurs (1938) ont montré que cette espèce était responsable de la transmission de la leishmaniose viscérale dans le foyer crétois.

La figure 5 schématise la densité (en nombre de phlébotomes pour 100 pièges) de $P$. major dans les différentes stations de notre itinéraire transect. Il apparaît nettement que cette espèce n'est abondante qu'au-dessus de $300 \mathrm{~m}$ et rare aux basses altitudes. Cette répartition correspond bien à ce que nous connaissons de certaines zones d'endémie leishmanienne dans le bassin méditerranéen (Serbie, Corse, Cévennes) où les foyers de leishmaniose viscérale se localisent à mi-pente des collines entre 300 et $600 \mathrm{~m}$, ou $800 \mathrm{~m}$ suivant les régions. Il est probable qu'ici comme en Crète, $P$. major est le principal vecteur. A noter que c'est la seule espèce que nous ayons trouvée dans un chenil ayant hébergé deux chiens leishmaniens.

\section{- Phlebotomus perfiliewi Parrot 1930:}

En ce qui concerne la Grèce cette espèce n'avait été signalée jusqu'ici que de Macédoine (Hertig, 1949).

Elle a été incriminée dans la transmission du Bouton d'Orient en Italie (Corradetti, 1952), du kala-azar en Serbie (Zivkovic, 1972) et de la leishmaniose canine en Tunisie (Dancesco et coll., 1970). Du fait de son abondance, son rôle ici, en association peut-être avec $P$. major, doit être envisagé. 


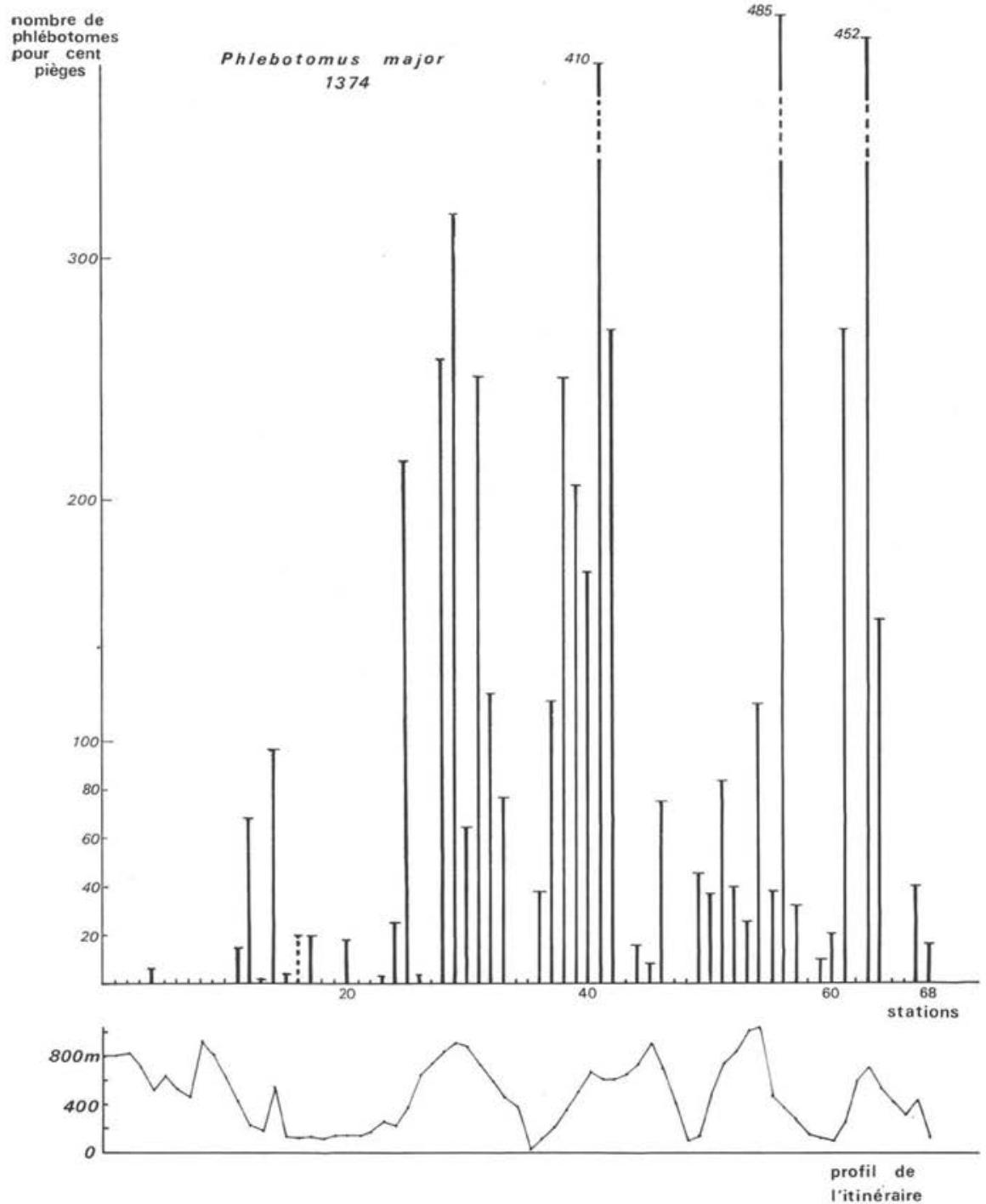

Fig. 5. - Répartition de $P$. major le long de l'itinéraire transect. 


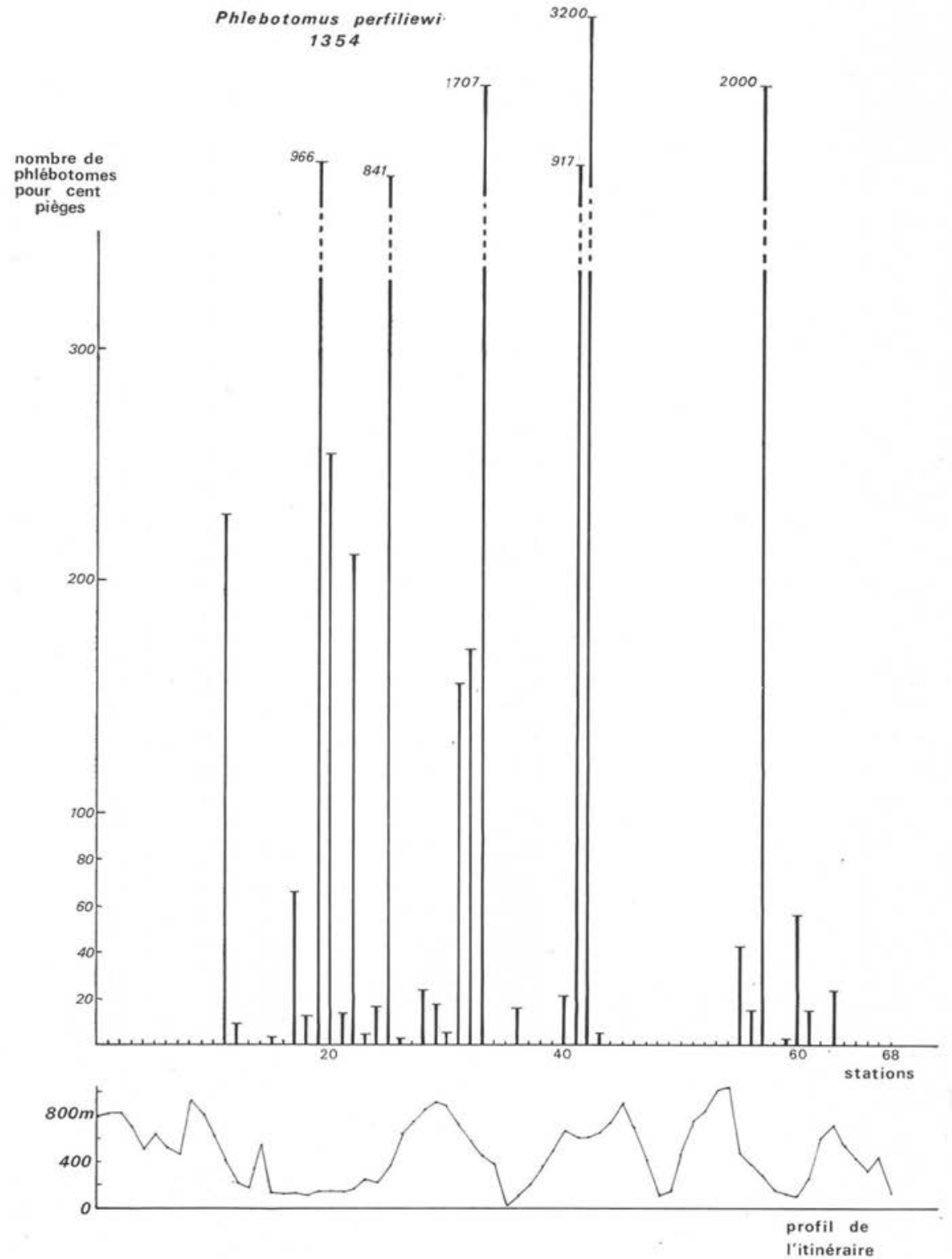

Fig. 6. - Répartition de $P$. perfiliewi le long de l'itinéraire transect. 
Sa répartition tout au long de notre itinéraire est hétérogène (fig. 6) et nous n'avons trouvé aucune corrélation $\mathrm{ni}$ avec l'altitude ni avec la nature du biotope: la forte concentration dans certaines stations correspond peut-être à la présence d'un hôte préférentiel.

- Phlebotomus tobbi Adler et Théodor 1930:

Ce Phlébotome a été signalé comme vecteur possible de leishmaniose viscérale (Lewis, 1974). A priori, il est présent ici en trop petit nombre pour pouvoir jouer un rôle efficace à moins qu'en accord avec les observations de Zivkovic et Miscevic (1973), il ne s'agisse d'une espèce moins précoce que les précédentes, susceptible d'entrer en scène plus tard dans la saison. En effet, elle représente un pourcentage non négligeable des captures effectuées de juin à septembre 1939 par Hadjinicolaou dans la région de Salonique. Mais elle est absente d'Athènes et de Crète (Hadjinicolaou, 1958).

\section{$4^{\circ}$ Sous-genre Adlerius :}

Plusieurs espèces appartenant à ce groupe sont des vecteurs potentiels de leishmaniose viscérale.

- Phlebotomus simici Nitzulescu 1931:

- et P. chinensis balcanicus Théodor 1958:

Hertig (1949) signale la présence en Grèce de $P$. chinensis simici que des auteurs autorisés (Théodor, 1958 ; Zivkovic, 1972) élèvent au rang d'espèce : $P$. simici.

En 1958, sur un unique exemplaire capturé à Jannitsa (Macédoine grecque), Théodor décrit $P$. chinensis balcanicus.

Dans le même temps Hadjinicolaou (1958) cite $P$. chinensis sans rien préciser.

Pour notre part, à côté de $P$. simici (fig. 7) nous avons trouvé en petit nombre (5 mâles et 5 femelles) des $P$. chinensis balcanicus Théodor 1958 typiques (fig. 8).

Que faut-il alors penser des « $P$. chinensis» d'Hadjinicolaou qui semblent très répandus dans la région de Salonique et qui par conséquent pourraient y jouer un rôle de vecteur efficace?

L'une d'entre nous (N. Léger) ayant eu l'occasion, grâce à l'obligeance du $\mathrm{P}^{r}$ Zivkovic de Belgrade, d'examiner des Phlébotomes du groupe chinensis en provenance de Macédoine yougoslave, a pu observer, à côté de $P$. chinensis balcanicus typiques, des exemplaires difficiles à classer du fait de la présence de caractères intermédiaires. Seule une révision de l'espèce, avec examen comparatif de préparations en provenance de Yougoslavie, de Grèce et de Roumanie (Dancesco, 1968), permettrait de clarifier la situation. 


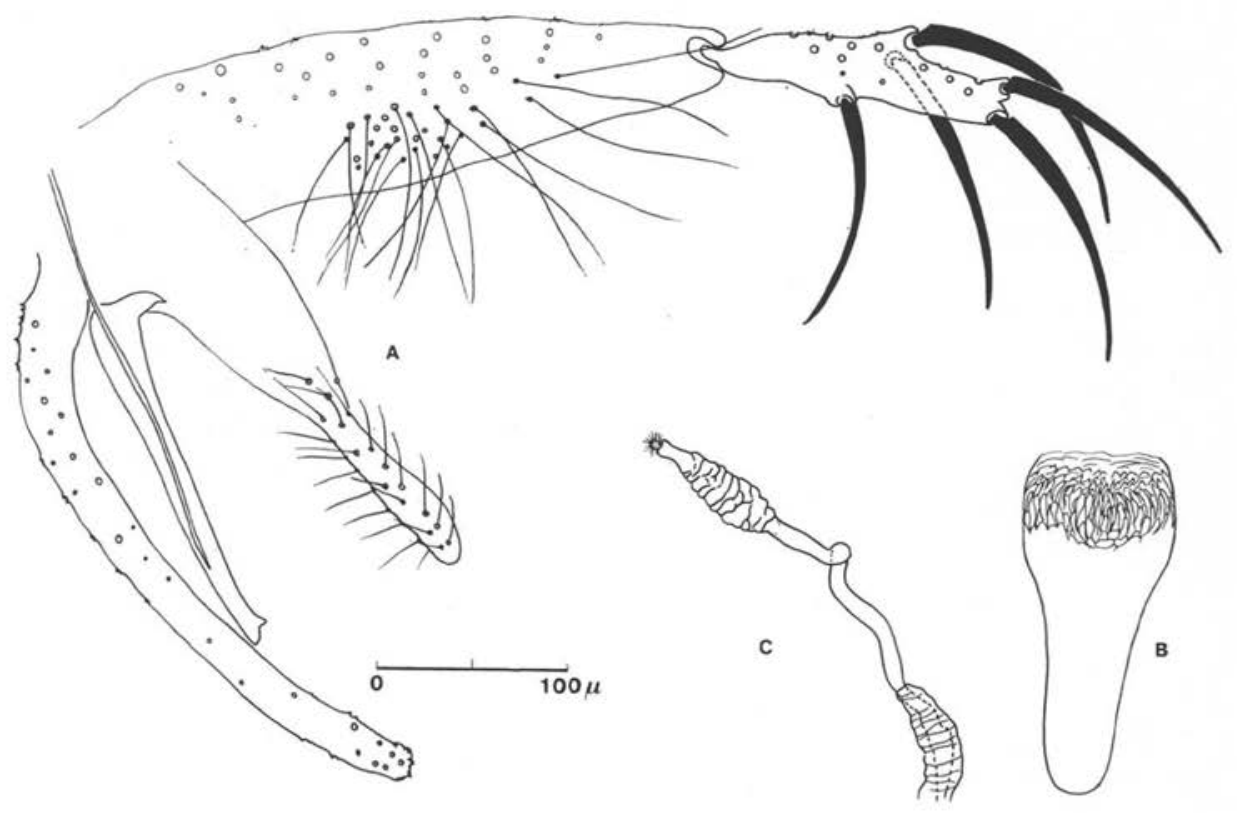

Fig. 7. - Phlebotomus simici $\delta^{\star}$ et $\uparrow . \mathrm{A}$, genitalia ; $\mathrm{B}$, pharynx $q$; $\mathrm{C}$, spermathèque.

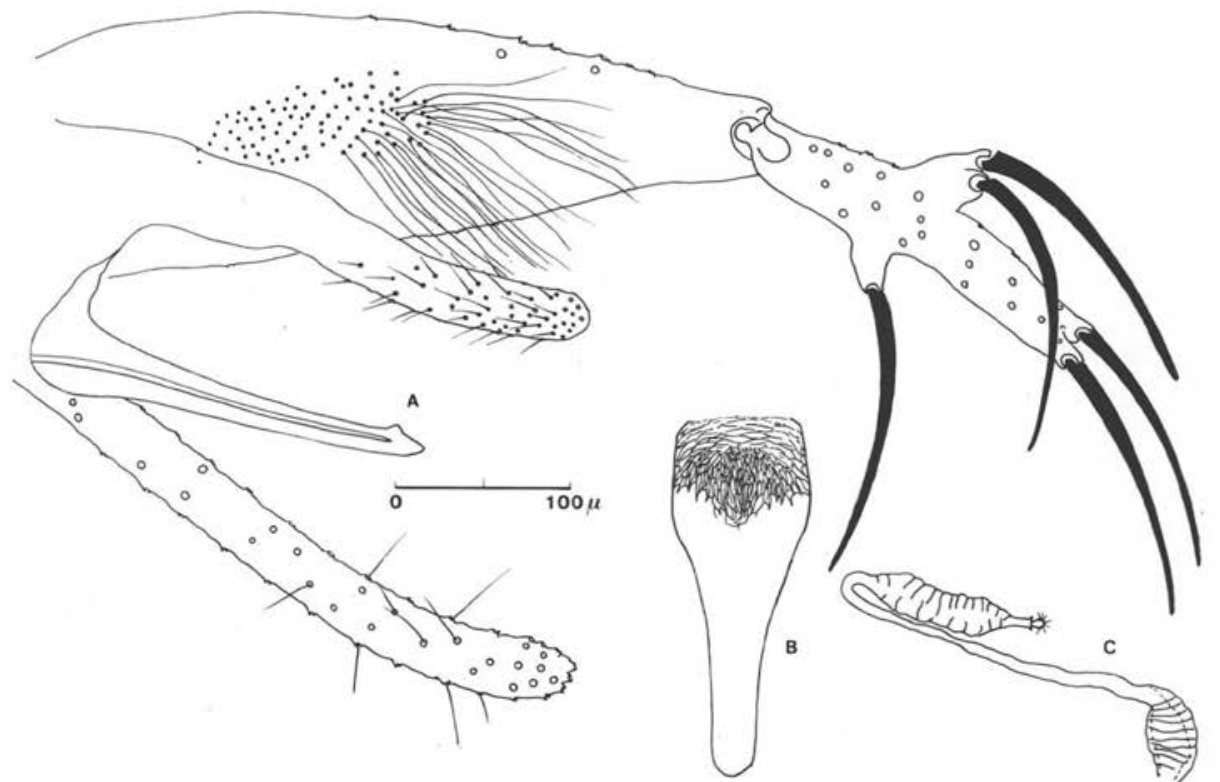

Fig. 8. - Phlebotomus chinensis balcanicus ot et $q$. A, genitalia; B, pharynx $q$; C, spermathèque. 


\section{B. - Genre Sergentomyia :}

- Sergentomyia minuta (Rondani, 1843):

La division de cette espèce en deux sous-espèces : $S$. $m$. minuta et $S$. $m$. parroti par Parrot (1929) et Théodor (1958), est basée sur l'importance de l'armature cibariale chez les femelles.

Nous avons montré dans un précédent travail (Rioux et coll., 1975) que dans la partie occidentale de son aire (France, Espagne, Maroc) l'espèce $S$. minuta se comporte comme un cline, à moins qu'il ne s'agisse, éventualité moins vraisemblable, d'hybridation entre deux sous-espèces géographiques. Au cours de ce travail la numération des dents cibariales a été effectuée sur 103 exemplaires femelles capturés le long de notre itinéraire transect, c'est-à-dire entre le $38^{\circ}$ et le $40^{\circ}$ degré de latitude nord (fig. 9).

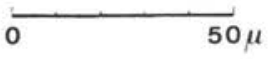

A

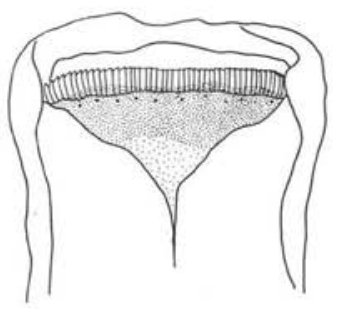

Sergentomyia minuta $€$
A

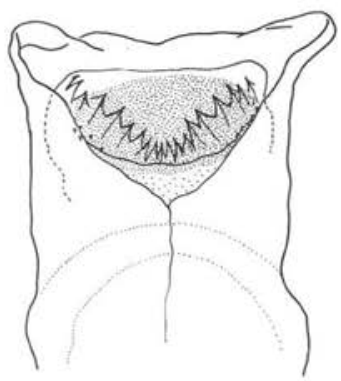

Sergentomyia dentata $q$

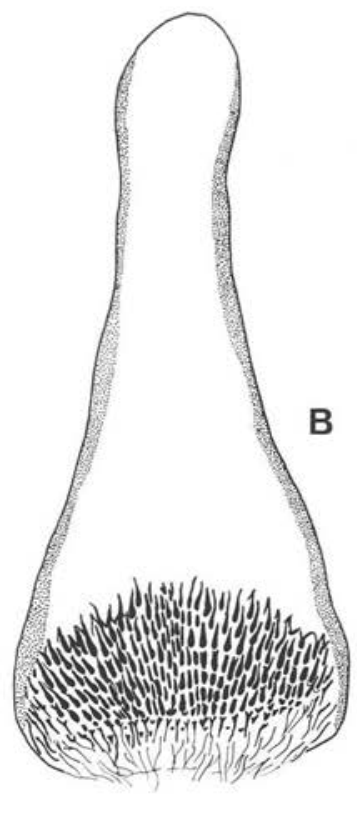

Fig. 9. - Sergentomyia minuta et Sergentomyia dentata †. A, cibarium; B, pharynx.

L'examen de la courbe de répartition du nombre de dents (fig. 10) et le calcul des différents paramètres, nous montre que la population examinée est tout à fait compara- 


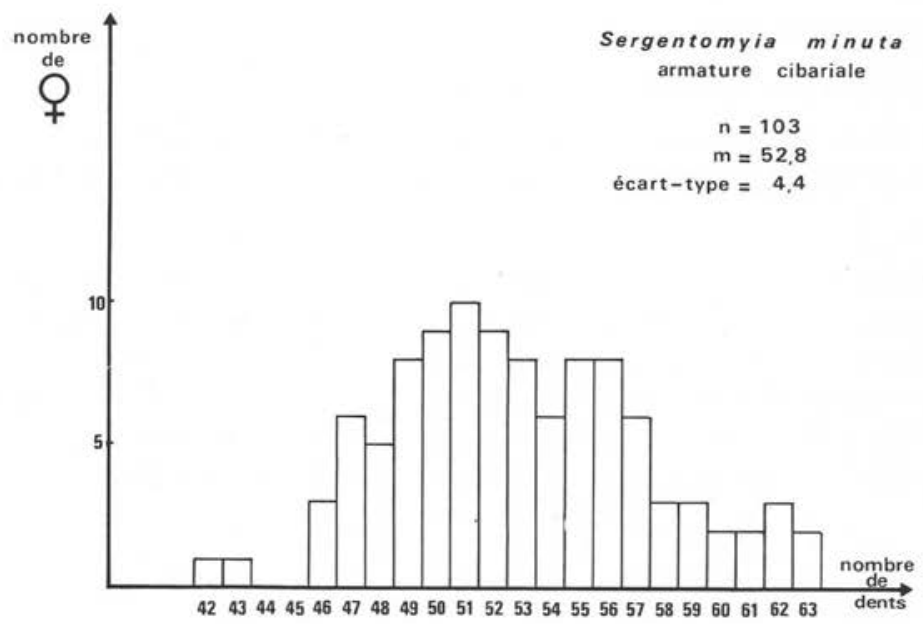

Fig. 10. - Importance de l'armature cibariale femelle dans les populations de Sergentomyia minuta récoltées.

ble à celle que nous avons eu l'occasion d'étudier en Espagne, à la même latitude : sur le diagramme comparatif publié précédemment, nos $S$. minuta de Grèce viennent s'insérer entre ceux de Chilches (Castellon) et ceux de Puerto Lumbreras (Murcia) (fig. 11).

Ceci vient à l'appui de l'existence d'un cline nord-sud de $S$. minuta et justifie l'emploi de la nomenclature binominale simple.

- Sergentomyia dentata (Sinton, 1933):

Dans nos captures c'est l'espèce la plus abondante et la plus ubiquiste (Tableau II).

Cette espèce a été subdivisée par divers auteurs, souvent sans doute de façon injustifiée. La réalité des diverses sous-espèces, voire espèces, serait à réenvisager comme nous avons eu l'occasion de le faire pour d'autres Sergentomyia (Léger et coll., 1974 ; Rioux et coll., 1975).

De ce groupe Hertig (1949) signale deux espèces présentes en Grèce : $S$. theodori Parrot, 1942 et $S$. bruchoni Parrot, 1935.

Hadjinicolaou (1958) n'en mentionne qu'une: $S$. theodori.

Nous rangeant à l'avis de Théodor (1958) et de Houin et coll. (1971) nous pensons que $S$. bruchoni est synonyme de $S$. dentata.

Le statut des $S$. theodori de Grèce serait également à revoir, l'absence de $S$. dentata (ou bruchoni) des captures d'Hadjinicolaou nous semblant sujette à caution.

Quoi qu'il en soit nous avons rangé nos Sergentomyia du groupe dentata, qui sont tous du même type (fig. 9 et 12) sous le binôme: Sergentomyia dentata considérant 


\section{Sergentomyia minuta}

armature cibariale

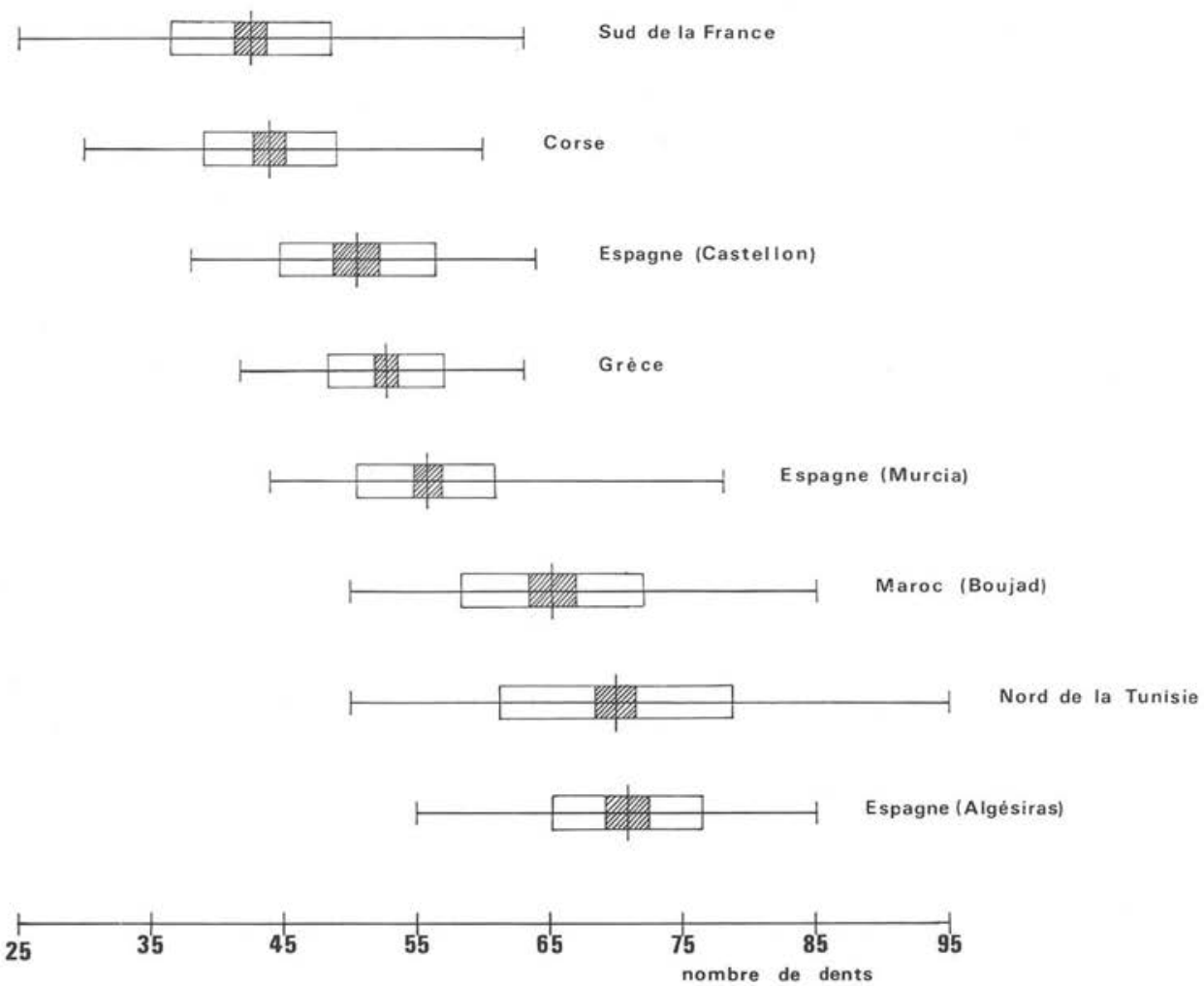

Fig. 11. - Le cline Sergentomyia minuta (Rondani 1843). Trait vertical: moyenne de l'échantillon. Rectangle hachuré : intervalle de confiance $(95 \%)$ de la moyenne. Rectangle blanc: écart-type. Ligne horizontale: extrêmes observés.

la synonymie avec $S$. bruchoni comme acquise et la forme du pharynx (fig. 9) excluant le diagnostic de $S$. theodori. Quant à son rôle éventuel dans l'épidémiologie des leishmanioses, nous ne pouvons qu'adhérer à l'opinion de Houin et coll. dans leur travail sur les Phlébotomes de Turquie (1971):

* A notre connaissance, cette espèce n'a jamais été signalée comme anthropophile, même occasionnelle, mais il serait du plus haut intérêt, compte tenu de son abondance et de son ubiquité dans la région considérée, d'étudier ses caractères éthologiques. » 


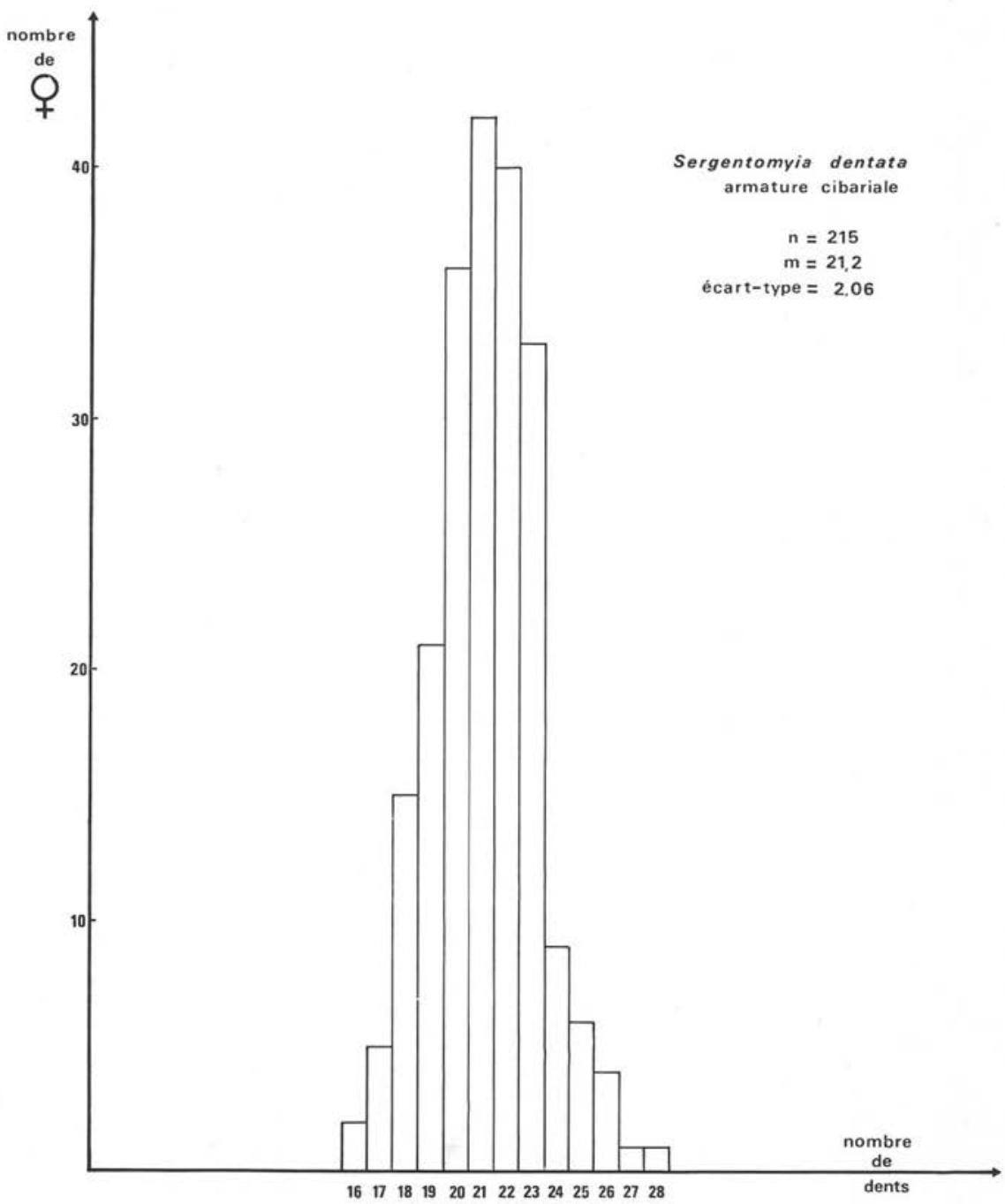

Fig. 12. - Importance de l'armature cibariale femelle dans les populations de Sergentomyia dentata récoltées.

\section{Bibliographie}

Adler (S.), Theodor (O.), 1931 : Investigations on Mediterranean kala-azar, I, Introduction and epidemiology. Proc. $R$. Soc. London, Ser. B, 108, 447-453.

Adler (S.), Theodor (O.), 1931 : Investigation on Mediterranean kala-azar, VIII, Further observations on Mediterranean sandflies. Proc. R. Soc. London, Ser. B, 116, 505-5.15. 
Adler (S.), Theodor (O.), Witenberg (G.), 1938: A study of leishmaniasis in Canea, Crete. Proc. R. Soc., Ser. B, 125, 491-516.

Caminopetros (J.), 1934 : Sur la faune des phlébotomes de la Grèce. Bull. Soc. Pathol. Exot., 27, 450-455.

Caminopetros (J.), 1935: Addition à la liste des phlébotomes signalés pour la première fois en Grèce. Bull. Soc. Pathol. Exot., 28, 44-46.

Corradetti (A.), 1952: Ricerche epidemiologiche sulla leishmaniosi cutanea in Abruzzo ed esperienze dirette alla totale interruptione della transmissione (1948-1950). R.C. Ist. Sup. San., 15, 181-187.

Croset (H.), Rioux (J.A.), Léger (N.), Houin (R.), Cadi Soussi (M.), Benmansour (N.), 1977: Les méthodes d'échantillonnage des populations de Phlébotomes en région méditerranéenne. In: Ecologie des leishmanioses, C.N.R.S., édit., Paris, publ. n 239, 139-151.

Dancesco (P.), 1968 : Données sur la sous-espèce Phlebotomus chinensis balcanicus Theodor 1958 (Diptera Psychodidae) en Roumanie. Arch. Inst. Pasteur Tunis, 45, 185-194.

Dancesco (P.), Dedet (J.P.), Ben Osman (F.), Chadli (A.), 1970: Les phlébotomes capturés dans les foyers de leishmaniose canine à Tunis. Rôle probable de $P$. perniciosus et $P$. perfiliewi dans la transmission. Arch. Inst. Pasteur Tunis, 47, 65-68.

Hadjinicolaou (J.), 1958: Present status of Phlebotomus in certain areas of Greece. Bull. Org. Mond. Santé, 19, 967-979.

Hertig (M.), 1949 : Phlebotomus and residual DDT in Greece and Italy. Am. J. Trop. Med., 29, 773-809.

Houin (R.), Abonnenc (E.), Deniau (M.), 1971: Phlébotomes du sud de la Turquie. Ann. Parasitol. Hum. Comp., 46, 633-652.

Léger (N.), Rioux (J.A.), Croset (H.), Cadi Soussi (M.), Benmansour (N.), 1974: Le complexe Sergentomyia (Sergentomyia) antennata (Newstead 1912). Remarques systématiques et écologiques à propos de 948 exemplaires récoltés dans le Sud-Marocain. Ann. Parasitol. Hum. Comp., 49, 577-591.

Lewis (D.J.), 1974: The biology of Phlebotomidae in relation to leishmaniasis. Annual Rev. Entomol., 69, 363-384.

Parrot (L.), 1929: Note sur les Phlébotomes. III 2. Morphologie et répartition géographique de Phlebotomus parroti Adler et Theodor. Arch. Inst. Pasteur Algérie, 7, 306-307.

Parrot (L.), 1935: Phlébotomes en Grèce. Arch. Inst. Pasteur Algérie, 13, 249-255.

Parrot (L.), 1936: Phlébotomes de Grèce. Arch. Inst. Pasteur Algérie, 14, 50-52.

Perfiliev (P.P.), 1966 : Faune de 1'U.R.S.S. Diptères, vol. III, n 2, Phlebotomidae. 1 vol., 382 pp., Acad. Sci. U.R.S.S., édit., Moscou-Léningrad. (Traduction anglaise 1968, Israel program for scientific translations, édit.).

Rioux (J.A.), Croset (H.), Léger (N.), Maistre (M.), 1975: Remarques sur la taxonomie infraspécifique de Sergentomyia minuta, Sergentomyia africana et Sergentomyia antennata. Ann. Parasitol. Hum. Comp., 50, 635-641.

Theodor (O.), 1958: Die Fliegen der Palaearktischen region, $9 c$ Psychodidae-Phlebotominae. 1 vol., 55 p., E. Schweizerbart'sche, edit., Stuttgart.

Zivkovic (V.), 1972: Etat actuel des Phlébotomes (Diptera, Psychodidae) dans quelques anciens foyers endémiques de leishmaniose viscérale en Yougoslavie. Rec. Trav. Inst. Recherches Méd., Belgrade, 25-33.

Zivkovic (V.), Miscevic (Z.), 1973: Sandflies (Diptera, Psychodidae) in human dwellings and animal quarters of the village of Azbresnica (région de Nis). Acta Veterinaria, Beograd, 23, $225-233$. 